\title{
Efusão uveal associada à tuberculose presumida: relato de caso
}

\author{
Uveal effusion associated with presumed tuberculosis: case report
}

\author{
Juliana Ribeiro Rodrigues ${ }^{1}$ \\ Amanda Vieira Abílio ${ }^{2}$ \\ Pedro Durães Serracarbassa ${ }^{3}$
}

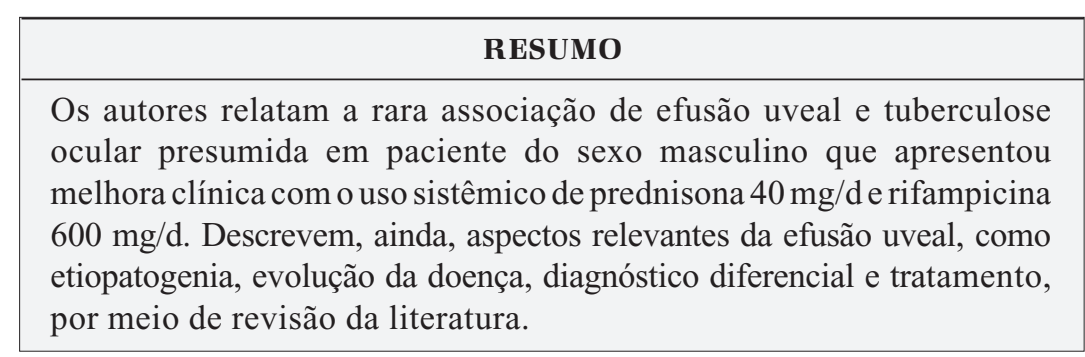

Descritores: Doenças da coróide; Doenças da úvea; Descolamento retiniano; Uveíte; Tuberculose ocular; Relatos de casos [Tipo de publicação]

\section{INTRODUÇÃO}

A efusão cílio-coroidiana é um acúmulo de material proteináceo em meio às fibras que ligam a coróide e o corpo ciliar à esclera. Classifica-se a efusão cílio-coroidiana ou efusão uveal como uma reação não específica observada em várias entidades clínicas ${ }^{(1)}$, podendo estar associada às doenças sistêmicas como Vogt-Koyanagi-Harada ${ }^{(1-2)}$; apresentar causas específicas como cirurgias oculares $^{(3)}$ ou pós-traumáticas ${ }^{(1)}$; quadros não inflamatórios como leucemias $^{(1)}$, mieloma múltiplo ${ }^{(1)}$, tumor de glândula lacrimal ${ }^{(1)}$, linfomas ${ }^{(4)}$, alterações vasculares (fístula carótido-cavernosa) ${ }^{(1)}$ ou surgir após fotocoagulação retiniana $^{(1)}$. O mixedema também é descrito como causa de efusão uveal $^{(5)}$. Quadros inflamatórios como episclerite e esclerite, pseudotumor orbitário, oftalmia simpática e doenças do colágeno também podem levar à efusão cílio-coroidiana ${ }^{(1)}$. Uveíte infecciosa como a sífilis ${ }^{(6)}$ e a toxoplasmose $^{(1)}$ também são causas importantes. A efusão uveal e o nanoftalmo são duas entidades reconhecidamente relacionadas ${ }^{(5,7)}$. A efusão uveal em olhos nanoftálmicos pode ocorrer de forma espontânea, após cirurgia de catarata, pós-trabeculoplastia; após cirurgia filtrante antiglaucomatosa e após laserterapia retiniana ${ }^{(8-9)}$.

Em alguns casos de efusão cílio-coroidiana, a causa não é aparente e não há associação com doenças sistêmicas, a esta chamamos de síndrome da efusão uveal idiopática que é semelhante à observada no nanoftalmo, exceto pelo diâmetro normal do globo ocular ${ }^{(1,10)}$.

No presente trabalho, apresenta-se um caso de efusão uveal secundária, sendo a tuberculose a etiologia mais provável.

Rodrigueso para correspondência: Juliana Ribeiro Paulo (SP) CEP 05409-011

E-mail: julianaoftalmo@gmail.com

Recebido para publicação em 08.03.2007

Última versão recebida em 16.07.2008

Aprovação em 01.08.2008

Nota Editorial: Depois de concluída a análise do artigo sob sigilo editorial e com a anuência do Dr. Haroldo Vieira de Moraes Jr. sobre a divulgação de seu nome como revisor, agradecemos sua participação neste processo.

\section{RELATO DO CASO}

O caso refere-se ao paciente J.P., de 50 anos de idade, sexo masculino, cor parda, natural de Luís, Estado de São Paulo. Procurou o ambulatório de oftalmologia queixando-se de baixa acuidade visual (BAV) de surgimento 
súbito no olho direito, com duração de 30 dias. A BAV é caracterizada pelo paciente como "uma mancha escura e central". Negava história de trauma, dor ou hiperemia ocular. O interrogatório clínico sobre os demais aparelhos foi negativo.

No exame oftalmológico observou-se refração dinâmica plana para longe e adição para perto de $+2,00$ em cada olho. Acuidade visual com correção era de 20/200 no olho direito e 20/20 no olho esquerdo. Ausência de alterações no exame de biomicroscopia anterior e da musculatura extrínseca ocular. Pressão ocular no momento da consulta: 8 e $12 \mathrm{mmHg}$ no olho direito e olho esquerdo respectivamente. Reflexos fotomotor direto e indireto presentes e normais em ambos os olhos.

No mapeamento de retina notou-se olho esquerdo (OE) sem alterações e olho direito (OD) com descolamento de retina seroso inferior, atingindo mácula e descolamento de coróide nos $360^{\circ}$ da periferia da retina (Figuras 1 A e B; 2 A e B).
Foi realizada angiofluoresceinografia (AGF); ultra-sonografia ocular (USG) e ultra-sonografia biomicroscópica (UBM) em ambos os olhos. À AGF notaram-se pontos de hiperfluorescência do tipo extravasamento do contraste, ao nível do EPR e no pólo posterior, que aumentaram de intensidade e tamanho com o decorrer do exame (Figuras 3 A a E). A USG ocular do olho direito denotou descolamento de vítreo parcial, espessamento de coróide e descolamento de retina na periferia (Figuras 4 A e B) e a UBM demonstrou espessamento (edema) e descolamento de corpo ciliar (Figuras $5 \mathrm{~A}$ a C).

Foram realizados exames subsidiários: hemograma com leucocitose e neutrofilia; IgG positivo para toxoplasmose, rubéola e mononucleose, PPD forte reator (enduração de $18 \mathrm{~mm}$ ), VDRL negativo e RX de tórax sem alterações.

O paciente foi considerado com forte suspeita para tuberculose ocular e recebeu rifampicina $600 \mathrm{mg} / \mathrm{d}$ e prednisona
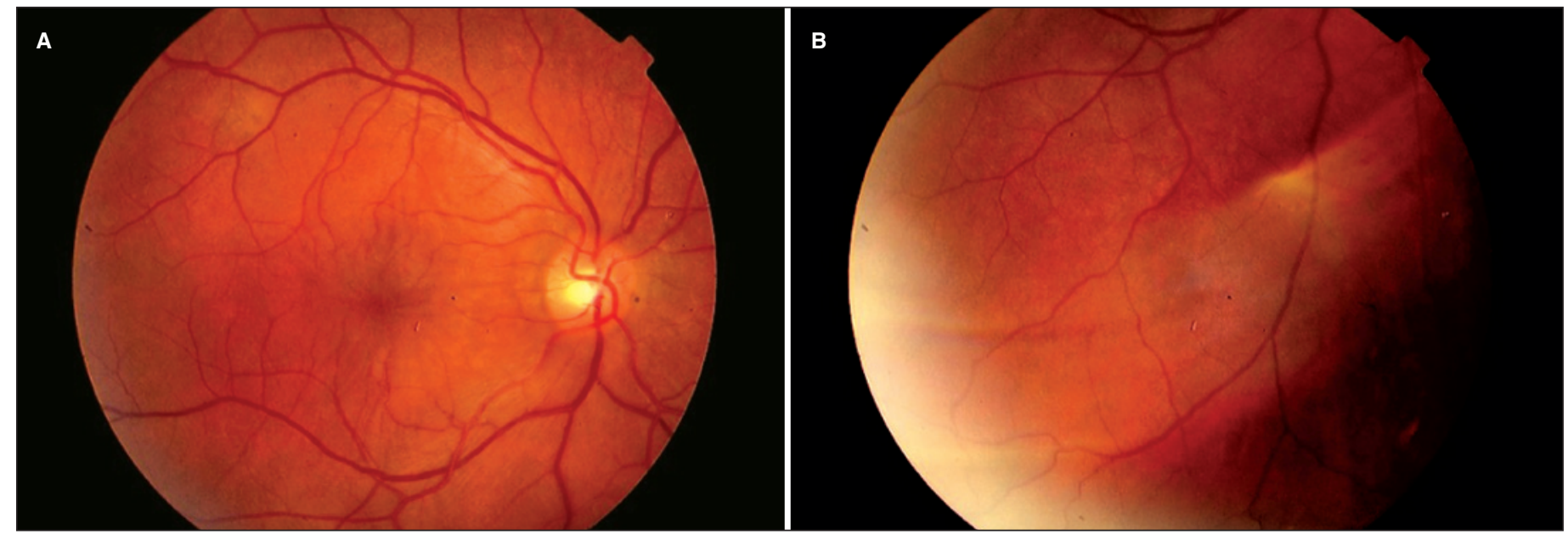

Figura 1 - Retinografia do olho direito: A) Descolamento seroso da mácula; B) Descolamento seroso de retina inferior e descolamento de coróide $360^{\circ}$, na periferia
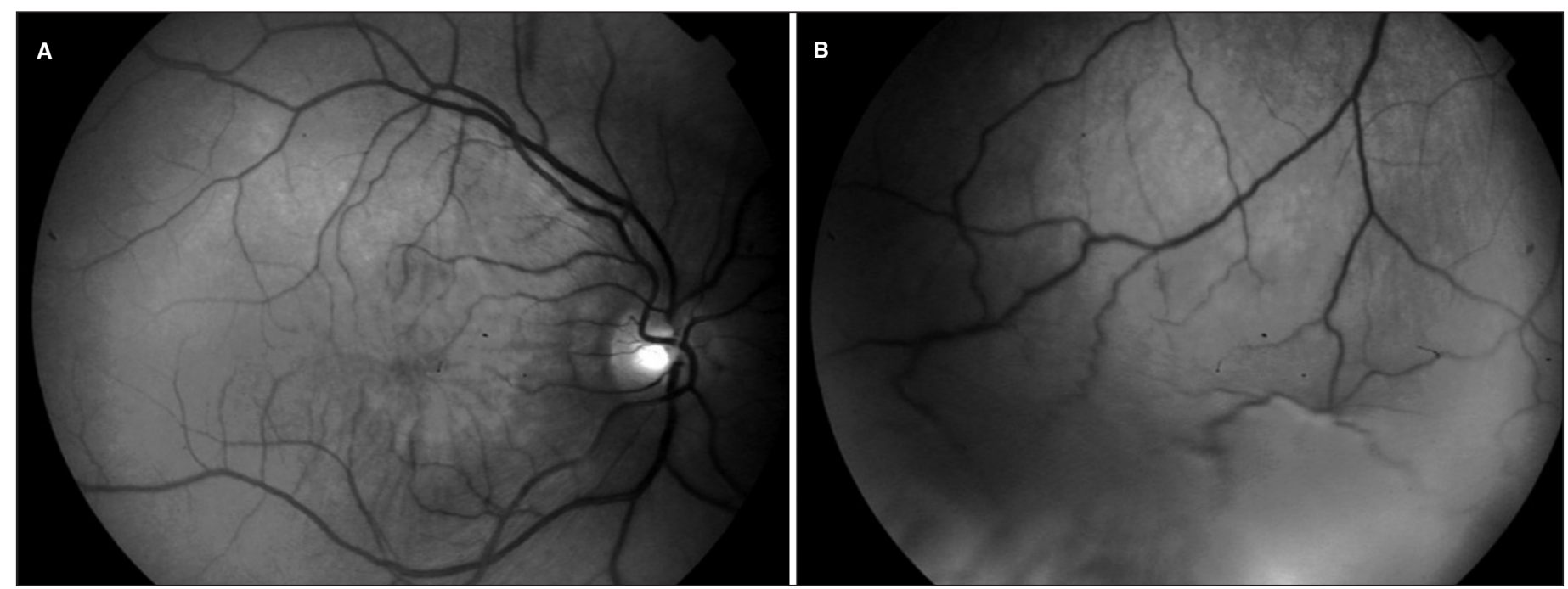

Figura 2 - Retinografia do olho direito: A) Foto aneritra demonstra o descolamento seroso da mácula; B) Foto aneritra demonstra o descolamento seroso de retina na região inferior 

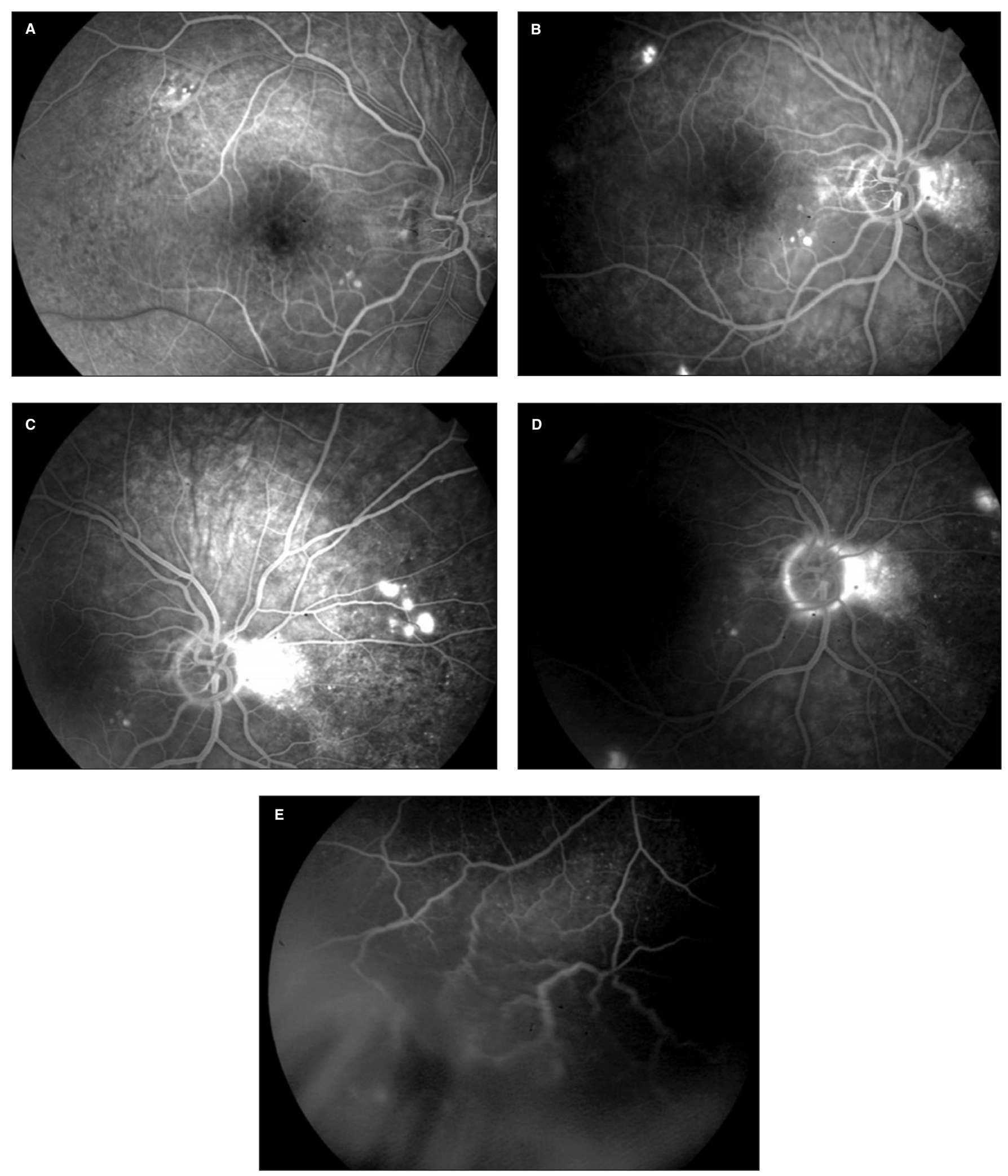

Figura 3 - Angiofluoresceinografia do olho direito: A) Fase inicial: pontos de hiperfluorescência, decorrentes do extravasamento do contraste, ao nível do EPR; B) Fase intermediária: hiperfluorescência puntiforme, ao nível de EPR, em pólo posterior; C) Pontos de vazamento do contraste nasal ao disco óptico; D) Fase tardia: pontos de hiperfluorescência que aumentaram em intensidade e tamanho com odecorrer do exame; E) Observa-se descolamento seroso de retina periférico 

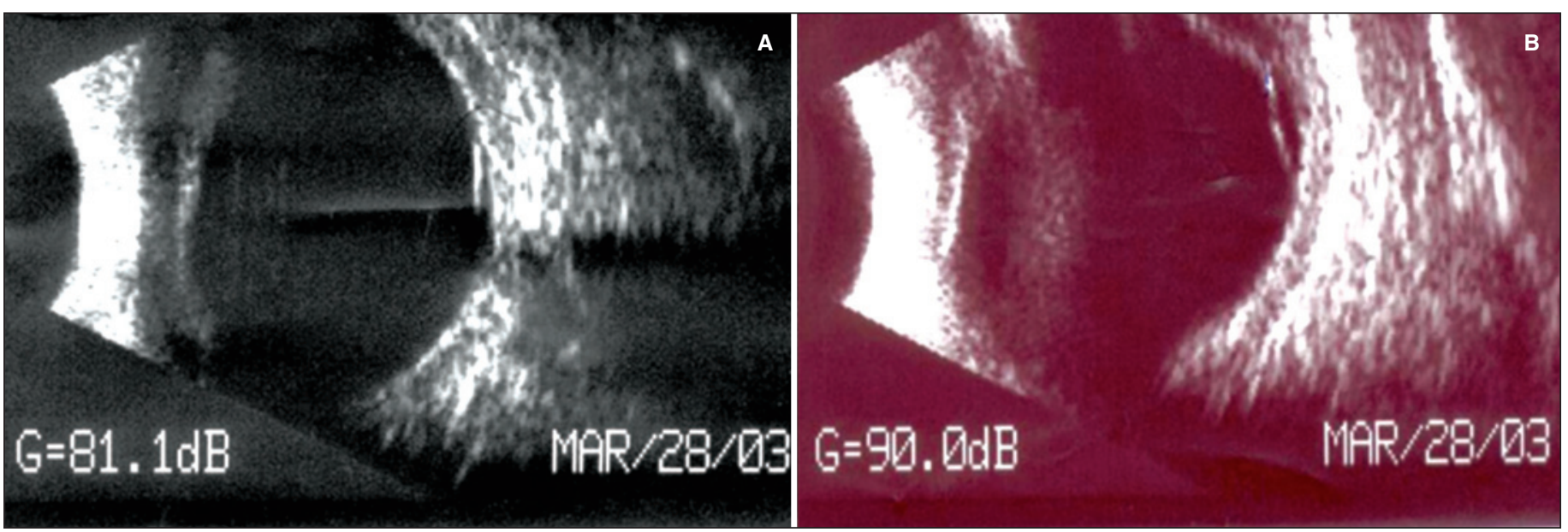

Figura 4 - Ultra-sonografia do olho direito: A) Presença de descolamento parcial do vítreo e espessamento da coróide; B) Evidência do descolamento de retina em periferia e do espessamento da coróide

$40 \mathrm{mg} / \mathrm{d}$ via oral. Após 30 dias do início do tratamento, houve melhora da acuidade visual no OD para 20/20 e resolução do descolamento de retina seroso, porém com descolamento de coróide presente. Nos três meses de seguimento, a acuidade visual ainda era de 20/20, a retina permanecia aplicada e o descolamento de coróide permanecia inalterado.

\section{DISCUSSÃO}

A efusão uveal pode ser idiopática ou de causas secundárias. A síndrome de efusão uveal idiopática foi primeiramente descrita por Schepens e Brockhurst em 1963, os autores descreveram 17 casos de síndrome de efusão uveal presentes, predominantemente, em homens de meia-idade, sendo que $30 \%$ deles apresentavam descolamento de coróide anular e o espessamento escleral foi encontrado em $47 \%$ do total dos pacientes $^{(11)}$.

O descolamento de coróide idiopático seroso, de corpo ciliar e de retina são descritos como síndrome de efusão uveal e, uma das causas dessa desordem são anormalidades esclerais que levam ao aumento da resistência ao fluxo transescleral de proteínas intra-oculares ${ }^{(12)}$. Esta teoria explica a causa da efusão uveal onde o espessamento escleral é evidente, mas falha em relação àquela onde a espessura escleral é normal. Acredita-se que neste último caso há alterações na permeabilidade vascular coroidiana levando à efusão $\mathrm{O}^{(13-14)}$.

A síndrome de efusão uveal idiopática acomete principalmente indivíduos saudáveis e homens de meia-idade ${ }^{(10,12)}$. A apresentação inicial mais comum é a baixa acuidade visual central, descolamento de retina exsudativo, acompanhado de espessamento ou descolamento de coróide e de corpo ciliar anular $^{(5,8,12)}$. Caracteriza-se também pela dilatação dos vasos episclerais e pressão ocular normal ${ }^{(12)}$. A angiofluoresceinografia desses olhos geralmente revela retardo da perfusão coroidiana, fluorescência coroidiana prolongada, áreas focais de hiperfluorescência por vazamento do contraste através do epitélio pigmentado da retina e acúmulos de pigmentos semelhantes à pele de leopardo que surgem após alguns meses do descolamento ${ }^{(10,12)}$. Alguns autores mostraram dois casos de efusão uveal idiopática nos quais a angiografia com indocianina verde apresentou alterações coroidianas sugestivas de inflamação inespecífica, caracterizadas por dilatação dos vasos e por áreas focais de hiperfluorescência perivascular coroidiana de início tardio, por extravasamento do contraste ${ }^{(14)}$. A USG ocular é importante no diagnóstico de descolamento de retina, descolamento de coróide e de corpo ciliar e informa espessura de esclera e diâmetro axial do globo ocular.

Através da imunohistoquímica e microscopia eletrônica, é possível demonstrar o acúmulo anormal de glicosaminoglicanas na esclera, o que prejudica o fluxo transescleral de fluídos e contribui para o espessamento escleral. Essas observações descritas sugerem que o defeito na síntese e degradação das proteoglicanas na esclera pode representar uma forma de mucopolissacaridose ocular ${ }^{(15)}$.

A doença tem um curso indolente, podendo levar meses a anos para a resolução do quadro. O tratamento clínico com corticoterapia e antimetabólitos muitas vezes é ineficaz ${ }^{(10,12)}$, sugerindo ausência de inflamação, sendo o tratamento cirúrgico muitas vezes necessário, como a descompressão das veias vorticosas, com sucesso limitado, e esclerotomias a fim de aumentar o fluxo de fluídos supracoroidais, obtendo assim algum sucesso no manejo dessa entidade.

Todas as entidades clínicas causadoras de efusão cíliocoroidiana secundária formam o diagnóstico diferencial da síndrome de efusão uveal idiopática, são elas: síndrome de VogtKoyanagi-Harada ${ }^{(2)}$; nanoftalmia ${ }^{(5,7)}$, tumores coroidianos (metástases, linfoma, melanoma) ${ }^{(1,4)} ;$ mixedema $^{(5)}$; esclerite $^{(1)}$; sífi$\operatorname{lis}^{(6)}$; toxoplasmose ${ }^{(1)}$; coroidorretinopatia serosa central idiopática $^{(12)}$; fístula carótido-cavernosa ${ }^{(1)}$; oftalmia simpática ${ }^{(1)} \mathrm{e}$ pseudotumor orbitário(1).

O presente estudo relata um caso de efusão uveal secundária, sendo a tuberculose ocular a etiologia mais provável, diagnosti- 


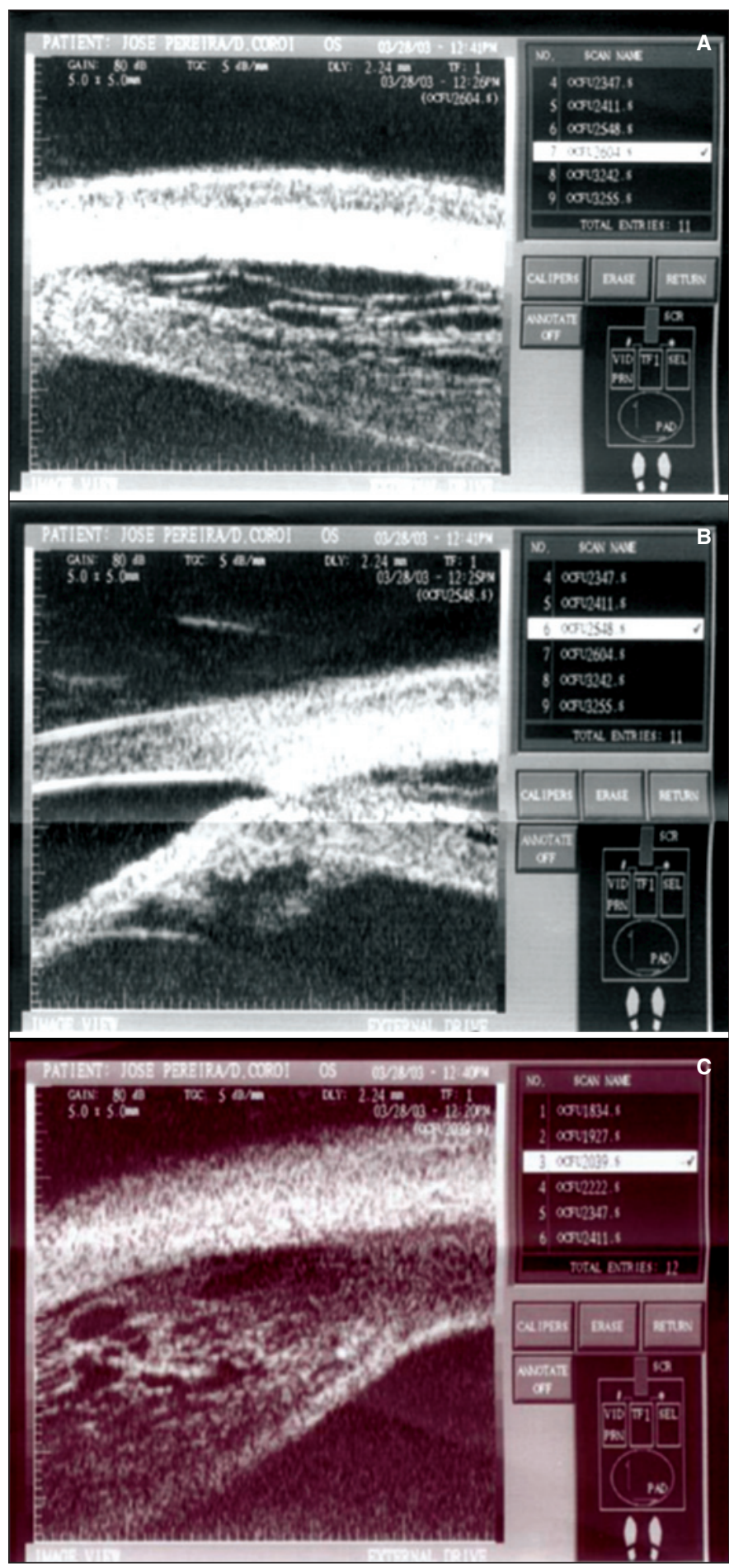

Figura 5 - Ultra-sonografia biomicroscópica do olho direito: A) Observase espessamento do corpo ciliar; B) Descolamento e edema do corpo ciliar; C) Espessamento (edema) do corpo ciliar

cada através da alteração ocular presente, ausência de doença sistêmica e PPD forte reator. Estudo prévio analisou 17 pacientes com diagnóstico de tuberculose ocular, encontrando como forma mais comum de apresentação a uveíte crônica bilateral granulomatosa ( $35,3 \%$ dos casos) sendo que, apesar da diversidade de apresentações clínicas, nenhum caso evoluiu com efusão uveal ${ }^{(16)}$. Nesse mesmo estudo a doença sistêmica era presente em $41,1 \%$ dos casos. O paciente do presente relato desconhecia contato com portadores de tuberculose, não tinha história pregressa da doença, nem diagnóstico de tuberculose extra-ocular na época, além de não possuir outras alterações oculares, exceto o descolamento de coróide e descolamento de retina exsudativo. Poucos são os diagnósticos de efusão uveal devido às uveítes, É descrito um caso de retinite por toxoplasmose com uveíte secundária, coroidite nodular e efusão cílio-coroidiana ${ }^{(1)}$.

Os exames complementares realizados e a clínica oftalmológica descartam as diversas causas de descolamento de retina exsudativo e efusão cílio-coroidiana, levando a uma forte suspeita de uma etiologia inflamatória e infecciosa para o caso, que é sustentada pela melhora do quadro clínico com o início do tratamento. É válida a possibilidade da melhora clínica ter ocorrido apenas pelo uso do corticóide que tem ação antiinflamatória bem conhecida, não ocorrendo influência da rifampicina administrada, por isso, considera-se como o diagnóstico mais provável para o presente caso a tuberculose ocular presumida.

\section{ABSTRACT}

The authors report a rare association of uveal effusion with presumed ocular tuberculosis in a male patient who presented clinical improvement with the systemic use of prednisone $40 \mathrm{mg} / \mathrm{d}$ and rifampicin $600 \mathrm{mg} / \mathrm{d}$. In addition, relevant aspects of the uveal effusion such as the pathogenesis, evolution of the disease, differential diagnosis and treatment are described through a revision of the literature.

Keywords: Choroid diseases; Uveal diseases; Retinal detachment; Uveitis; Tuberculosis, ocular; Case reports [Publication type]

\section{REFERÊNCIAS}

1. Green WR, Kincaid MC, Fogle JA. Uveal effusion syndrome. Trans Ophthal Soc UK. 1981;101(Pt 3):368-75.

2. Damico FM, Kiss S. Young LH. Vogt-Koyanagi-Harada disease. Semin Ophthalmol. 2005;20(3):183-90. Review.

3. Sabti K, Lindley SK, Mansour M, Discepola M. Uveal effusion after cataract surgery: an echographic study. Ophthalmology. 2001;108(1):100-3.

4. Gaucher D, Bodaghi B, Charlotte F, Schneider C, Cassoux N, Lemaitre C, et al [MALT-type B-cell lymphoma masquerading as scleritis or posterior uveitis] J Fr Ophtalmol. 2005;28(1):31-8. French.

5. Ward RC, Gragoudas ES, Pon DM, Albert DM. Abnormal scleral findings in uveal effusion syndrome. Am J Ophthalmol. 1988;106(2):139-46.

6. DeLuise VP, Clark SW $3^{\text {rd }}$, Smith JL. Syphilitic retinal detachment and uveal effusion. Am J Ophthalmol. 1982;94(6):757-61.

7. Han L, Cairns JD. Nanophthalmos with longstanding choroidal effusion and serous retinal detachment. Aust N Z J Ophthalmol. 1997;25(2):181-3.

8. Bhagat N, Lim JL, Minckler DS, Green RL. Posterior uveal effusion syndrome after trabeculectomy in an eye with ocular venous congestion. Br J Ophthalmol. 2004;88(1):153-4

9. Lesnoni G, Rossi T, Nistri A, Bocassini B. Nanophthalmic uveal effusion syndrome after prophylactic laser treatment. Eur J Ophthalmol. 1999;9(4):315-8.

10. Anand R, Tasman WS. Nonrhegmatogenous retinal detachment. In: Ryan SJ, editor. Retina. California: Mosby; 2001. p.2076-99. 
11. Schepens CL, Brockhurst RJ. Uveal effusion. I. Clinical Picture. Arch Ophthalmol. 1963;70:189-201.

12. Gass JD, Jallow S. Idiopatic serous detachment of the choroid, ciliary body, and retina (uveal effusion syndrome). Ophthalmology. 1982;89(9):1018-32.

13. Gass JD. Uveal effusion syndrome: a new hypothesis concerning pathogenesis and technique of surgical treatment, 1983. Retina. 2003(6 Suppl):159-63.
14. Kumar A, Kedar S, Singh RP. The Indocyanine green findings in idiopatic uveal effusion syndrome. Indian J Ophthalmol. 2002;50(3):217-9.

15. Forrester JV, Lee WR, Kerr PR, Dua HS. The uveal effusion syndrome and transscleral flow. Eye. 1990;4(Pt 2):354-65.

16. Almeida SRA, Finamor LP, Muccioli C. Alterações oculares em pacientes com tuberculose. Arq Bras Oftalmol. 2006;69(2):177-9.

\title{
III FÓRUM NACIONAL DE SAÚDE OCULAR
}

\author{
Brasilia - 30 de outubro de 2008
}

A Mello Faro Turismo, agência de viagens oficial para o evento, oferece em condições e preços especiais, a hospedagem em Brasília e o transporte aéreo a partir das principais cidades brasileiras.

\begin{tabular}{|c|c|c|c|c|}
\hline \multicolumn{2}{|c|}{ HOSPEDAGEM em BRASÍLIA } & $\begin{array}{l}\text { Diária do } \mathrm{Apt}^{\circ} \\
\text { PARA } 01 \text { PESSOA }\end{array}$ & $\begin{array}{c}\text { Diária do } \mathrm{Apt}^{\circ} \\
\text { PARA } 02 \text { PESSOAS }\end{array}$ & $\begin{array}{c}\text { Diária do } \text { Apt }^{\circ} \\
\text { PARA } 03 \text { PESSOAS }\end{array}$ \\
\hline $\begin{array}{l}\text { Bonaparte Suítes } \\
\text { SHS Quadra } 2 \text { Bloco J }\end{array}$ & Flat Primeira Superior & $\begin{array}{c}\mathbf{R} \$ \mathbf{2 9 9 , 0 0} \\
+10 \% \text { taxa }\end{array}$ & $\begin{array}{c}\mathbf{R} \$ \mathbf{3 4 4 , 0 0} \\
+10 \% \text { taxa }\end{array}$ & $\begin{array}{c}\mathbf{R} \$ \mathbf{3 8 9 , 0 0} \\
+10 \% \text { taxa }\end{array}$ \\
\hline $\begin{array}{l}\text { Mercure } \\
\text { SHN Quadra } 5 \text { Bloco G }\end{array}$ & Primeira & $\begin{array}{c}\text { R\$206,00 } \\
+5 \% \text { ISS } \\
\text { sem café da manhă }\end{array}$ & $\begin{array}{c}\mathbf{R} \$ \mathbf{2 4 1 , 0 0} \\
+5 \% \text { ISS } \\
\text { sem café da manhă }\end{array}$ & 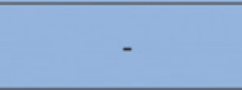 \\
\hline $\begin{array}{l}\text { Sonesta } \\
\text { SHN Quadra } 5 \text { Bloco B }\end{array}$ & Turística & $\begin{array}{l}\text { R\$ } 175,00 \\
+5 \% \text { ISS }\end{array}$ & $\begin{array}{l}R \$ 175,00 \\
+5 \% \text { ISS }\end{array}$ & - \\
\hline
\end{tabular}

TRANSPORTE AÉREO

Serão aplicadas tarifas promocionais segundo disponibilidade por ocasião da reserva e descontos especiais para o evento. Os bilhetes serão emitidos na forma de ordem de passagem ou bilhete eletrônico e estarão disponíveis na cidade de origem para pagamento parcelado por cartões de crédito segundo condições vigentes na época. A disponibilidade de vôos e tarifas serão informada durante o atendimento.

Outras informações e reserva diretamente

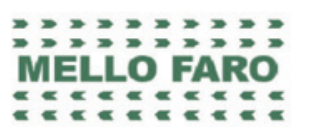

Av. São Luis, $165-1 \square$ andar - conj. $01^{\wedge}$ Exclusivo para o FÓRUM! Desconto de até $17 \%$ sobre as tarifas disponiveis no ato da reserva e emissão através da Mello Faro PAGAMENTO PARCELADO

Solicite a sua reserva diretamente pelo nosso site em ambiente seguro, ou preencha e envie por fax ou correio a Ficha de Reserva abaixo

\begin{tabular}{|c|c|c|c|c|c|}
\hline III FÓRUM & & & & \multicolumn{2}{|c|}{ FICHA DE RESERVA } \\
\hline Nome & \multicolumn{2}{|l|}{ Tel } & \multicolumn{3}{|c|}{ 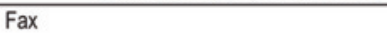 } \\
\hline Endereço & \multicolumn{2}{|l|}{ Cidade } & UF & Cep & \\
\hline \multirow[t]{2}{*}{ Acompanhante } & \multicolumn{5}{|l|}{ Email } \\
\hline & Chegada (dd/mm/aa) & Saida (dd/mm/aa) & & $\mathrm{N}^{0}$ noites & $\mathrm{N}^{0}$ pessoas \\
\hline
\end{tabular}

Solicito a reserva de hospedagem conforme a seguir: Disponibilidade limitada para ocupação em TRIPLO

Bonaparte $\square$ Mercure $\square$ Sonesta

encaminho em anexocheque ou comprovante de depósito de uma diária + taxas no hotel escolhido como $1^{\text {a }}$ opção;

autorizo a garantia da reserva com o meu cartão de crédito a seguir indicado assim como o débito correspondente a uma diária + taxas em caso do meu não comparecimento ao hotel no primeiro dia da reserva ou cancelamento após 10/10/2008.

Depósitos a favor da Mello Faro no Bradesco, Agência 095-7, Conta corrente 151.216-1 - CNPJ 65.838.427/0001-09 $\square$ Amex $\square$ Mastercard $\square$ Visa $\mathrm{N}^{\circ}$ : Cod. Segurança*: Portador:

* Atenção: é necessário informar o código de segurança do cartão (3 números após o número do cartão impresso na tarja de assinatura para Mastercard, Visa e quatro números gravados na frente e à direita para o Amex)

\section{PARA SUA PRÓPRIA SEGURANÇA SERÁ NECESSÁRIO O ENVIO POR FAX DE CÓPIA FRENTE I VERSO DO CARTÃO DE CRÉDITO}

Data___ 2008 